International Journal of Pure and Applied Mathematics

Volume 91 No. 3 2014, 403-420

ISSN: 1311-8080 (printed version); ISSN: 1314-3395 (on-line version)

url: http://www.ijpam.eu

doi: http://dx.doi.org/10.12732/ijpam.v91i3.15

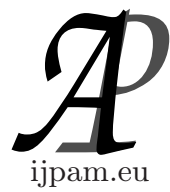

\title{
THE NEW WELL-POSEDNESS OF DISCONTINUOUS RIEMANN-HILBERT PROBLEM FOR ELLIPTIC COMPLEX EQUATIONS OF FIRST ORDER WITH TWO DEGENERATE LINES
}

\author{
G.C. Wen ${ }^{1} \S$, Y.H. Zhang ${ }^{2}$ \\ ${ }^{1}$ LMAM, School of Mathematical Sciences \\ Peking University \\ Beijing, 100871, P.R. CHINA \\ ${ }^{2}$ Department of Mathematics \\ Beijing Technology and Business University \\ Beijing, 100048, P.R. CHINA
}

\begin{abstract}
This article deals with some elliptic complex equations of first order, i.e. the generalized Beltrimi equation with two degenerate lines in the discussed multiply connected domain. We first propose the new well-posed-ness of discontinuous Riemman-Hilbert problem, give estimates of solutions for the modified boundary value problem. Afterwards by using the method of parameter extension, the existence of continuous solutions for the generalized Beltrimi equation is verified. In the article, the proof of Hölder continuity of a singular double integer is very difficult and interesting. The above problem possesses the important applications to the Tricomi problem of mixed type equations of second order.
\end{abstract}

AMS Subject Classification: 35J60, 35J70, 35J56

Key Words: elliptic complex equations, two degenerate lines, the new posedness of discontinuous Riemann-Hilbert problem, unique solvability of continuous solutions, Hölder continuity of singular integer

Received: December 3, 2013

(C) 2014 Academic Publications, Ltd.

${ }^{\S}$ Correspondence author url: www.acadpubl.eu 


\section{Formulation of Riemann-Hilbert Problem for (1.3) with Two Degenerate Lines}

Let $D$ be an $(N+1)$-connected domain with the boundary $\Gamma=\cup_{j=0}^{N} \Gamma_{j}$ in $\mathbb{C}$, where $\Gamma \in C_{\mu}^{1}(0<\mu<1)$. Without loss of generality, we assume that $D$ is a circular domain in $|z|<1$, bounded by the $(N+1)$-circles $\Gamma_{j}:\left|z-z_{j}\right|=$ $r_{j}, j=0,1, \ldots, N$ and $\Gamma_{0}=\Gamma_{N+1}:|z|=1, z=0 \in D$. The authors of [1-9] discussed some special cases of the continuous Riemann-Hilbert problem in $D$, in this paper the notations are the same as in References [7-18].

Consider the linear elliptic systems of first order equations with two degenerate lines as follows

$$
\left\{\begin{array}{l}
H_{3}(x) H_{2}(y) u_{x}-H_{1}(x) H_{4}(y) v_{y}=a_{1} u+b_{1} v+c_{1}, \\
H_{3}(x) H_{2}(y) v_{x}+H_{1}(x) H_{4}(y) u_{y}=a_{1} u+b_{2} v+c_{2},
\end{array} \text { in } D,\right.
$$

in which $H_{j}(x)=|x|^{m_{j} / 2} h_{j}(x), j=1,3, H_{j}(y)=|y|^{m_{j} / 2} h_{j}(y), j=2,4, h_{j}(x)(j$ $=1,3,), h_{j}(y)(j=2,4)$ are positive continuous functions in $\bar{D}, m_{j}(j=1,2)$ are positive constants with the condition $m=m_{1} /\left(1+m_{1}-m_{3}\right)+m_{2} /(1+$ $\left.m_{2}-m_{4}\right)<1$, and $a_{j}, b_{j}, c_{j}(j=1,2)$ are functions of $x+i y(\in D)$ satisfying the conditions $a, b, c, d \in L_{\infty}(D)$. The above conditions will be called conditions $a, b, c, d \in L_{\infty}(D)$. The above conditions will be called Condition C. From the elliptic condition in (1.1.2), Chapter I, [18], we have

$$
J=4 K_{1} K_{4}-\left(K_{2}+K_{3}\right)^{2}=4\left[H_{1}(x) H_{2}(y) H_{3}(x) H_{4}(y)\right]^{2}>0 \text { in } \bar{D} \backslash \gamma
$$

and $J=0$ on $\gamma=\{x=0,-1<y<1\} \cup\{-1<x<1, y=0\}$, hence system (1.1) is elliptic system of first order equations in $D \backslash \gamma$ and is parabolic degenerate on $\gamma=\gamma_{1} \cup \gamma_{2}$.

We shall write the complex form of the system (1.1) is as follows. Note that: 


$$
\begin{aligned}
& w=u+i v, w_{\bar{z}}=\left[w_{x}+i w_{y}\right] / 2, w_{z}=\left[w_{x}-i w_{y}\right] / 2, \\
& \bar{w}_{\bar{z}}=\left[\bar{w}_{x}+i \bar{w}_{y}\right] / 2, \bar{w}_{z}=\left[\bar{w}_{x}-i \bar{w}_{y}\right] / 2, \\
& (u+i v)_{x}=w_{x}=w_{\bar{z}}+w_{z},(u-i v)_{x}=\bar{w}_{x}=\bar{w}_{\bar{z}}+\bar{w}_{z}, \\
& 2 u_{x}=w_{\bar{z}}+w_{z}+\bar{w}_{\bar{z}}+\bar{w}_{z}, 2 i v_{x}=w_{\bar{z}}+w_{z}-\bar{w}_{\bar{z}}-\bar{w}_{z}, \\
& i(u+i v)_{y}=i w_{y}=w_{\bar{z}}-w_{z}, i(u-i v)_{y}=i \bar{w}_{y}=\bar{w}_{\bar{z}}-\bar{w}_{z}, \\
& 2 i u_{y}=w_{\bar{z}}-w_{z}+\bar{w}_{\bar{z}}-\bar{w}_{z},-2 v_{y}=w_{\bar{z}}-w_{z}-\bar{w}_{\bar{z}}+\bar{w}_{z}, \\
& H_{3}(x) H_{2}(y) u_{x}=H_{3}(x) H_{2}(y)\left[w_{\bar{z}}+w_{z}+\bar{w}_{\bar{z}}+\bar{w}_{z}\right] / 2, \\
& H_{1}(x) H_{4}(y) u_{y}=-i H_{1}(x) H_{4}(y)\left[w_{\bar{z}}-w_{z}+\bar{w}_{\bar{z}}-\bar{w}_{z}\right] / 2 \text {, } \\
& H_{3}(x) H_{2}(y) v_{x}=-i H_{3}(x) H_{2}(y)\left[w_{\bar{z}}+w_{z}-\bar{w}_{\bar{z}}-\bar{w}_{z}\right] / 2 \text {, } \\
& -H_{1}(x) H_{4}(y) v_{y}=H_{1}(x) H_{4}(y)\left[w_{\bar{z}}-w_{z}-\bar{w}_{\bar{z}}+\bar{w}_{z}\right] / 2, \\
& 2\left[H_{3}(x) H_{2}(y) u_{x}-H_{1}(x) H_{4}(y) v_{y}\right]=\left[H_{3}(x) H_{2}(y)+H_{1}(x) H_{4}(y)\right]\left[w_{\bar{z}}+\bar{w}_{z}\right] \\
& +\left[H_{3}(x) H_{2}(y)-H_{1}(x) H_{4}(y)\right]\left[w_{z}+\bar{w}_{\bar{z}}\right]=a_{1}(w+\bar{w})-i b_{1}(w-\bar{w})+2 c_{1}, \\
& 2\left[H_{3}(x) H_{2}(y) v_{x}+H_{1}(x) H_{4}(y) u_{y}\right]=-i\left[H_{3}(x) H_{2}(y)+H_{1}(x) H_{4}(y)\right]\left[w_{\bar{z}}-\bar{w}_{z}\right] \\
& -i\left[H_{3}(x) H_{2}(y)-H_{1}(x) H_{4}(y)\right]\left[w_{z}-\bar{w}_{\bar{z}}\right]=a_{2}(w+\bar{w})-i b_{2}(w-\bar{w})+2 c_{2}, \\
& w_{\bar{z}}=Q(z) w_{z}+A_{1}(z) w+A_{2}(z) \bar{w}+A_{3}(z), \\
& Q(z)=\left(H_{1}(x) H_{4}(y)-H_{3}(x) H_{2}(y)\right) /\left(H_{1}(x) H_{4}(y)+H_{3}(x) H_{2}(y)\right), \\
& A_{1}(z)=\left(a_{1}+a_{2} i-i b_{1}+b_{2}\right) /\left(H_{1}(x) H_{4}(y)+H_{3}(x) H_{2}(y)\right), \\
& \left.A_{2}(z)=\left(a_{1}+a_{2} i+i b_{1}-b_{2}\right)\right] /\left(H_{1}(x) H_{4}(y)+H_{3}(x) H_{2}(y)\right),= \\
& A_{3}(z)=2\left(c_{1}+j c_{2}\right) /\left(H_{1}(x) H_{4}(y)+H_{3}(x) H_{2}(y)\right) \text { in } D,
\end{aligned}
$$

and

$$
L_{\infty}\left[A_{j}(z)\left(H_{1}(x) H_{4}(y)+H_{3}(x) H_{2}(y)\right), \bar{D}\right] \leq k_{1}, j=1,2,3,
$$

where $k_{1}$ are positive constants.

In particular if $A_{1}(Z)=A_{2}(Z)=A_{3}(Z)=0$ in $D$, then the complex equation (1.3) is reduced to the Beltrami equation

$$
W_{\bar{z}}=Q(z) w_{z} \text { in } G \text {. }
$$

Now we formulate a new posed-ness of discontinuous boundary value problems as follows: 
Problem A. Find a continuous solution $w(z)$ of $(1.3)$ in $D$, which satisfies the boundary conditions

$$
\operatorname{Re}[\overline{\lambda(z)} w(z)]=r(z)+h(z), z \in \Gamma
$$

where $\lambda(z)(|\lambda(z)|=1), c(z)$ satisfy the conditions

$$
C_{\alpha}\left[\lambda(z), \hat{\Gamma}_{j}\right] \leq k_{0}, C_{\alpha}\left[\left|z-t_{j-1}\right|^{\beta_{j-1}}\left|z-t_{j}\right|^{\beta_{j}} c(z), \hat{\Gamma}_{j}\right] \leq k_{2}, j=1, \ldots, m,
$$

in which $\alpha(0<\alpha<1), k_{0}, k_{2}\left(\geq k_{0}\right)$ are positive constants; and

$$
\frac{h(z) \lambda(z)}{\tilde{X}(z)}=\left\{\begin{array}{l}
h_{j}, z \in \Gamma_{j}, j=1, \ldots, N, \\
0, z \in \Gamma_{0}, \\
h_{j}, z \in \Gamma_{j}, j=1, \ldots, N \\
{\left[1+(-1)^{2 K}\right] h_{0}+\operatorname{Re} \sum_{m=1}^{[\mid K+1 / 2]-1}\left(h_{m}^{+}+i h_{m}^{-}\right) z^{m}, z \in \Gamma_{0},}
\end{array}\right\} \text { if } K<0,
$$

in which $h_{j}\left(j=\left[1-(-1)^{2 K}\right] / 2, \ldots, N\right), h_{m}^{ \pm}(m=1, \ldots,-K-1, K<0)$ are unknown real constants to be determined appropriately, in which

$$
\begin{gathered}
K=\frac{1}{2 \pi} \Delta_{\Gamma_{j}} \arg \lambda(z)=\sum_{j=0}^{N} K_{j}, K_{j}=\left[\frac{\phi_{j}}{\pi}\right]+J_{j}, \quad J_{j}=0 \text { or } 1, \quad j=1, \ldots, m, \\
e^{i \phi_{j}}=\frac{\lambda\left(t_{j}-0\right)}{\lambda\left(t_{j}+0\right)}, \gamma_{j}=\frac{1}{\pi i} \ln \frac{\lambda\left(t_{j}-0\right)}{\lambda\left(t_{j}+0\right)}=\frac{\phi_{j}}{\pi}-K_{j},
\end{gathered}
$$

is called the index of Problem A, in which $0 \leq \gamma_{j}<1$ when $J_{j}=0$, and $-1<\gamma_{j}<0$ when $J_{j}=1, j=1, \ldots, m$, and $Z=\left\{t_{1}, \ldots, t_{m}\right\}$ are the first kind of discontinuous points of $\lambda(z)$ on $\Gamma, \hat{\Gamma}_{j}$ is an arc from the point $t_{j-1}$ to $t_{j}$ on $\Gamma$, and does not include the end point $t_{j}(j=1,2, \ldots, m)$, we can assume that $t_{j} \in \Gamma_{0}\left(j=1, \ldots, m_{0}\right), t_{j} \in \Gamma_{1}\left(j=m_{0}+1, \ldots, m_{1}\right), \ldots, t_{j} \in \Gamma_{N}(j=$ $\left.m_{N-1}+1 \ldots, m\right)$ are all discontinuous points of $\lambda(z)$ on $\Gamma$; there is no harm in assuming that the partial indexes $K_{l}$ of $\lambda(z)$ on $\Gamma_{l}\left(l=1, \ldots, N_{0}(\leq N)\right.$ are not integers, and the partial indexes $K_{l}$ of $\lambda(z)$ on $\Gamma_{l}\left(j=0, N_{0}+1, \ldots, N\right)$ are integers; $\beta_{j}\left(0<\beta_{j}<1\right), \gamma_{j}(j=1, \ldots, m)$ satisfy the conditions

$$
\beta_{j}+\left|\gamma_{j}\right|<1, j=1, \ldots, m \text {. }
$$

Denote by $\lambda\left(t_{j}-0\right)$ and $\lambda\left(t_{j}+0\right)$ the left limit and right limit of $\lambda(t)$ as 
$t \rightarrow t_{j}(j=1,2, \ldots, m)$ on $\Gamma$, and denote

$$
\begin{array}{r}
Y(z)=\prod_{j=1}^{m_{0}}\left(z-t_{j}\right)^{\gamma_{j}} \prod_{l=l}^{N}\left(z-z_{l}\right)^{-\left[\tilde{K}_{l}\right]} \prod_{j=m_{0}+1}^{m_{1}}\left(\frac{z-t_{j}}{z-z_{1}}\right)^{\gamma_{j}}\left(\frac{z-t_{1}^{\prime}}{z-z_{1}}\right) \\
\times \prod_{j=m_{N_{0}-1}+1}^{m_{N_{0}}}\left(\frac{z-t_{j}}{z-z_{N_{0}}}\right)^{\gamma_{j}}\left(\frac{z-t_{N_{0}}^{\prime}}{z-z_{N_{0}}}\right)_{j=m_{N_{0}}+1}^{m_{N_{0}+1}}\left(\frac{z-t_{j}}{z-z_{N_{0}+1}}\right)^{\gamma_{j}} \cdots \prod_{j=m_{N-1}+1}^{m}\left(\frac{z-t_{j}}{z-z_{N}}\right)^{\gamma_{j}},
\end{array}
$$

where $\tilde{K}_{l}=\sum_{j=m_{l-1}+1}^{m_{l}} K_{j}$ are denoted the partial index on $\Gamma_{l}(l=1, \ldots, N)$, $t_{l}^{\prime}\left(\in \Gamma_{l}, l=, \ldots, N_{0}\right)$ are fixed points, which are not the discontinuous points at $Z$; we must give the attention that the boundary circles $\Gamma_{j}(j=0,1, \ldots, N)$ of the domain $D$ are moved round the positive direct. Similarly to (1.7)-(1.12), Chapter V, [8], we see that

$$
\frac{\lambda\left(t_{j}-0\right)}{\lambda\left(t_{j}+0\right)} \overline{\left[\frac{Y\left(t_{j}-0\right)}{Y\left(t_{j}+0\right)}\right]}=\frac{\lambda\left(t_{j}-0\right)}{\lambda\left(t_{j}+0\right)} e^{-i \pi \gamma_{j}}= \pm 1, j=1, \ldots, m,
$$

it only needs to charge the symbol on some arcs on $\Gamma$, then $\lambda(z) \overline{Y(z)} /|Y(z)|$ on $\Gamma$ is continuous. In this case, its index

$$
\kappa=\frac{1}{2 \pi} \Delta_{\Gamma}[\lambda(z) \overline{Y(z)}]=K-\frac{N_{0}}{2}
$$

is an integer; and

$$
\begin{gathered}
\tilde{X}(z)=\left\{\begin{array}{l}
i z^{[\kappa]} e^{i S(z)} Y(z), z \in \Gamma_{0}, \\
i e^{i \theta_{j}} e^{i S(z)} Y(z), z \in \Gamma_{j}, j=1, \ldots, N,
\end{array} \quad \operatorname{Im}[\overline{\lambda(z)} \tilde{X}(z)]=0, z \in \Gamma,\right. \\
\operatorname{Re} S(z)=\left\{\begin{array}{l}
\arg \lambda(z)-[\kappa] \arg z-\arg Y(z), z \in \Gamma_{0}, \\
\arg \lambda(z)-\arg Y(z)-\theta_{j}, z \in \Gamma_{j}, j=1, \ldots, N,
\end{array} \quad \operatorname{Im}[S(1)]=0,\right.
\end{gathered}
$$

in which $S(z)$ is a solution of the modified Dirichlet problem with the above boundary condition for analytic functions, $\theta_{j}(j=1, \ldots, N)$ are real constants, and $\kappa=K-N_{0} / 2$. In addition the solution $w(z)$ is assumed to satisfy the point conditions

$$
\operatorname{Im}\left[\overline{\lambda\left(a_{j}\right)} w\left(a_{j}\right)\right]=b_{j}, j \in J=\{1, \ldots, 2 K+1\} \text { if } K \geq 0,
$$

where $a_{j} \in \Gamma_{0}(j=1, \ldots, 2 K+1, K \geq 0)$ are distinct points, and $b_{j}(j \in J)$ are all real constants with the constants $\left|b_{j}\right| \leq k_{3}(<\infty)$. 


\section{Equivalent Problem of Riemann-Hilbert Problem for (1.3)}

Now we give the another form of first order elliptic system (1.1). Setting

$$
\begin{aligned}
& X=X(x)=\int_{0}^{x}\left[H_{1}(t) / H_{3}(t)\right] d t,|X| \leq\left|s^{-2 / m_{1}} x\right|^{m_{1}-m_{3}+1}, \\
& Y=Y(y)=\int_{0}^{y}\left[H_{2}(t) / H_{4}(t)\right] d t,|Y| \leq\left|s^{-2 / m_{2}} y\right|^{m_{2}-m_{4}+1}, \\
& H_{1}(x)=|x|^{m_{1}} h_{1}(x) \geq(1 / s)|x|^{m_{1}}, H_{2}(y)=\int_{0}^{y} H_{2}(t) d t \geq(1 / s)|y|^{m_{2}},
\end{aligned}
$$

where $Z=X(x)+i Y(y)$ in $\bar{D}$, and $s$ is a sufficiently large positive constant, thus we have $H_{1}(x) \geq\left. s_{X}\right|^{m_{1} /\left(m_{1}-m_{3}+1\right)}, H_{2}(y) \geq s|Y|^{m_{2} /\left(m_{2}-m_{4}+1\right)}$. Denote

$$
\begin{gathered}
W(z)=u+i v, W_{\overline{\tilde{z}}}=\frac{1}{2}\left[H_{3}(x) H_{2}(y) W_{x}+i H_{1}(x) H_{4}(y) W_{y}\right] \\
=\frac{H_{1}(x) H_{2}(y)}{2}\left[W_{X}+i W_{Y}\right]=H_{1}(x) H_{2}(y) W_{X-i Y}=H_{1}(y) H_{2}(x) W_{\bar{Z}},
\end{gathered}
$$

where $d X=\left[H_{1}(x) / H_{3}(x)\right] d x, d Y=\left[H_{2}(y) / H_{4}(y)\right] d y$, then the system (1.1) can be written in the complex form then the system (2.1) can be written in the complex form

$$
\begin{gathered}
W_{\overline{\tilde{z}}}=H_{1}(x) H_{2}(y) W_{\bar{Z}}=A(Z) W+B(Z) \bar{W}+C(Z) \text { in } G, \\
A(Z)=\frac{1}{4}\left[a_{1}+i a_{2}-i b_{1}+b_{2}\right], B(Z)=\frac{1}{4}\left[a_{1}+i a_{2}+i b_{1}-b_{2}\right], \\
C(Z)=\frac{1}{2}\left(c_{1}+i c_{2}\right),
\end{gathered}
$$

in which $G$ is the image domain of $D$ with respect to the mapping $Z=Z(z)=$ $X(x)+i Y(y)$ in $D, z=z(Z)$ is the inverse function of $Z=Z(z)$, and $A(Z), B(Z), C(Z)$ satisfy

$$
L_{\infty}[A(Z), \bar{G}], L_{\infty}[B(Z), \bar{G}] \leq k_{0}, L_{\infty}[C(Z), \bar{G}] \leq k_{1},
$$

where $k_{0}, k_{1}$ are positive constants.

In particular if $A(Z)=B(Z)=C(Z)=0$ in $G$, then the complex equation $(2.3)$ is reduced to the simple complex equation

$$
W_{\bar{Z}}=Q(Z) W_{Z} \text { in } G \text {. }
$$

Introduce the corresponding modified Riemann-Hilbert boundary condition for the equation (2.4) as follows: 
Problem B. Find a continuous solution $w(Z)$ of (2.3) in $\bar{D}$ satisfying the boundary conditions

$$
\operatorname{Re}[\overline{\Lambda(Z)} W(Z)]=R(Z)+H(Z), z \in \hat{\Gamma},
$$

in which

$$
\begin{aligned}
& \frac{H(Z) \Lambda(Z)}{\tilde{X}[z(Z)]}= \\
& =\left\{\begin{array}{ll}
h_{j}, Z \in \hat{\Gamma}_{j}=Z\left(\Gamma_{j}\right), j=1, \ldots, N, \\
0, Z \in \hat{\Gamma}_{0}, & \text { if } K \geq 0, \\
h_{j}, Z \in \hat{\Gamma}_{j}, j=1, \ldots, N, \\
{\left[1+(-1)^{2 K}\right] h_{0}+\operatorname{Re} \sum_{m=1}^{[|K|+1 / 2]-1}\left(h_{m}^{+}+i h_{m}^{-}\right)[z(Z)]^{m}, z \in \hat{\Gamma}_{0},}
\end{array}\right\} \text { if } K<0,
\end{aligned}
$$

where $|\Lambda(Z)|=1, \hat{\Gamma}=Z(\Gamma), \hat{\Gamma}_{j}=Z\left(\Gamma_{j}\right)(j=0,1, \ldots, N), h_{j}\left(j=\left[1-(-1)^{2 K}\right] / 2\right.$, $\ldots, N), h_{m}^{ \pm}(m=1, \ldots,-K-1, K<0)$ are undetermined real constants. Moreover for $K \geq 0$ the solution $W(Z)$ is assumed to satisfy the point conditions

$$
\operatorname{Im}\left[\overline{\Lambda\left(\hat{a}_{j}\right)} W\left(\hat{a}_{j}\right)\right]=B_{j}, j \in J=\{1, \ldots, 2 K+1\},
$$

in which $\hat{a}_{j}=Z\left(a_{j}\right) \in \hat{\Gamma}_{0}(j=1, \ldots, 2 K+1, K \geq 0)$ are distinct points, and $B_{j}(j \in J)$ are all real constants, where $K=\frac{1}{2 \pi} \Delta_{\hat{\Gamma}} \arg \Lambda(z)$ is called the index of $\Lambda(Z)$ on $\hat{\Gamma}$. The coefficients in (2.6) and (2.7) satisfy the conditions

$$
C_{\alpha}[\Lambda(Z), \hat{\Gamma}] \leq k_{0}, C_{\alpha}[R(Z), \hat{\Gamma}] \leq k_{2},\left|B_{j}\right| \leq k_{2}, j \in J,
$$

in which $\alpha(0<\alpha<1), k_{0}, k_{2}\left(\geq k_{0}\right)$ are positive constants. Under Condition $\mathrm{C}$, we can find the unique solution $W(Z)$ of Problem B for equation (2.3) in $G$.

Theorem 2.1. Problem B for the equation (2.3) is solvable if and only if Problem A for (1.3) is solvable.

Proof. If we find a solution $W(Z)$ of Problem B for (2.3), and $W(Z)$ satisfies the boundary conditions

$$
\begin{aligned}
& \operatorname{Re}[\overline{\Lambda(Z)} W(Z)]=R(Z)+H(Z), Z \in \hat{\Gamma}, \\
& \operatorname{Im}\left[\overline{\lambda\left(\hat{a}_{j}\right)} W\left(\hat{a}_{j}\right)\right]=B_{j}, j \in J .
\end{aligned}
$$

then the solution $w(z)=W[Z(z)]$ is a requirement solution of Problem A, where $\lambda(z)=\Lambda[Z(z)], r(z)=R[Z(z)], h(Z)=H[Z(z)]$ and $b_{j}=B_{j}, a_{j}=z\left(\hat{a}_{j}\right), j \in$ $J$. 
Inversely we find any solution $w(z)$ of Problem A for (1.3), which satisfies the boundary conditions

$$
\begin{gathered}
\operatorname{Re}[\overline{\lambda(z)} w(z)]=r(z)+h(z), z \in \Gamma, \\
\operatorname{Im}\left[\overline{\lambda\left(a_{j}\right)} w\left(a_{j}\right)\right]=b_{j}, j \in J,
\end{gathered}
$$

where $\tilde{a}_{j}=Z\left(a_{j}\right), j \in J$, in which $z=z(Z)$ is the inverse function of $Z=$ $Z(z)=X+j Y$, then $W(Z)=w[z(Z)]$ is a requirement solution of Problem B satisfying the boundary conditions

$$
\operatorname{Re}[\overline{\Lambda(z)} W(Z)]=R(Z)+H(Z), Z=Z(z) \in \hat{\Gamma}, \operatorname{Im}\left[\overline{\Lambda\left(\hat{a}_{j}\right)} W\left(\hat{a}_{j}\right)\right]=B_{j}, j \in J
$$

where $\Lambda(Z)=\lambda[z(Z)], R(Z)=r[z(Z)], H(Z)=h[z(Z)], a_{j}=z\left(\hat{a}_{j}\right), b_{j}=$ $B_{j}, j \in J$, it is just our requirement.

\section{Solvability of Riemann-Hilbert Problem (Problem B) for (2.4)}

Theorem 3.1. Suppose that Problem B of the complex equation (2.3) satisfies Condition C. Then any solution $W(Z)$ of Problem B for (2.3) satisfies the estimates

$$
C_{\beta}[W(Z), \bar{G}] \leq M_{1}=M_{1}(k, \beta, D)
$$

in which $k=k\left(k_{0}, k_{1}, k_{2}\right), \beta(0<\beta<\alpha)$ and $M_{1}$ is a positive constant only dependent on $k, \beta, D$.

Proof. On the basis of Theorem 1.1.3, Chapter I, [18], the solution $W(Z)$ of Problem B can be expressed the formula

$$
\begin{gathered}
W(z)=\Phi(Z) e^{\phi(Z)}+\psi(Z) \\
\phi(Z)=T g=-\frac{1}{\pi} \iint_{D} \frac{g(\zeta)}{H_{1}(\operatorname{Re} z(\zeta)) H_{2}(\operatorname{Im} z(\zeta))(\zeta-Z)} d \sigma_{\zeta} \text { in } D
\end{gathered}
$$

in which $\Phi(Z)$ is an analytic function and $g(Z)=A(Z)+B(Z) \overline{W(Z)} / W(Z)$, $\psi(Z)=T f$ in $G$, hence the boundary value problem (Problem B) can be transformed into the boundary value problem (Problem $\tilde{B}$ ) for analytic functions

$$
\operatorname{Re}[\overline{\tilde{\Lambda}(Z)} \Phi(Z)]=\tilde{R}(Z)+\hat{H}(Z), z \in \hat{\Gamma}
$$




$$
\begin{aligned}
& \frac{\hat{H}(Z) \lambda[z(Z)]}{\tilde{X}[z(Z)]}= \\
& =\left\{\begin{array}{l}
0, Z \in \hat{\Gamma}_{0}, \\
h_{j}, Z \in \hat{\Gamma}_{j}, j=1, \cdots, N, \\
h_{j}, Z \in \hat{\Gamma}_{j}, j=1, \cdots, N \\
{\left[1+(-1)^{2 K}\right] h_{0}+\operatorname{Re} \sum_{m=1}^{[|K|+1 / 2]-1}\left(h_{m}^{+}+i h_{m}^{-}\right)[z(Z)]^{m}, z \in \hat{\Gamma}_{0},}
\end{array}\right\} \text { if } K \geq 0 \\
& \operatorname{Im}\left[\overline{\tilde{\Lambda}\left(\hat{a}_{j}\right)} \Phi\left(\hat{a}_{j}\right)\right]=\tilde{B}_{j}, j \in J
\end{aligned}
$$

where

$$
\begin{aligned}
& \overline{\tilde{\Lambda}(Z)}=\overline{\lambda(Z)} e^{\phi(Z)]}, \tilde{R}(Z)=R(Z)-\operatorname{Re}\{\overline{\tilde{\Lambda}(z)} \psi(Z)\}, \\
& \tilde{B}_{j}=B_{j}-\operatorname{Im}\left[\overline{\Lambda\left(\hat{a}_{j}\right)} \psi\left(\hat{a}_{j}\right)\right], j \in J .
\end{aligned}
$$

It can be seen that $\tilde{\Lambda}(Z), \tilde{R}(Z), \tilde{B}_{j}(j \in J)$ satisfy the conditions

$$
C_{\eta \beta}[\tilde{\Lambda}(Z), \hat{\Gamma}] \leq M_{3}, C_{\eta \beta}[\tilde{R}(Z), \hat{\Gamma}] \leq M_{2},\left|\tilde{B}_{j}\right| \leq M_{3}, j \in J,
$$

in which $M_{j}=M_{j}(k, \beta, D), j=2,3$. If we can prove that the solution $\Phi(\zeta)$ of Problem B satisfies the estimate

$$
C_{\eta \beta}[\Phi(Z), \bar{G}] \leq M_{4},
$$

in which $M_{4}=M_{4}(k, \beta, D)$, then by the method in the proof of Theorem 3.23.4, Chapter V, [8], the estimates (3.7) can be verified.

It remains to prove that (3.7) holds. For this, we first verify the boundedness of $\Phi(\zeta)$, i.e.

$$
C[\Phi(Z), \overline{\hat{G}}] \leq M_{5}=M_{5}(k, \beta, D) .
$$

Suppose that (3.8) is not true. Then there exist sequences of functions $\left\{\tilde{\Lambda}_{n}(Z)\right\}$, $\left\{\tilde{R}_{n}(Z)\right\},\left\{\tilde{B}_{j n}\right\}$ satisfying the same conditions as $\tilde{\Lambda}(Z), \tilde{R}(Z), \tilde{B}_{j}$, which uniformly converge to $\tilde{\Lambda}_{0}(z), \tilde{R}_{0}(Z) \tilde{B}_{j 0}(j \in J)$ on $\Gamma$ respectively. For the solution $\Phi_{n}(Z)$ of the boundary value problem (Problem $B_{\mathrm{n}}$ ) corresponding to $\tilde{\Lambda}_{n}(Z), \tilde{R}_{n}(Z), \tilde{B}_{j n}(j \in J)$, we have $I_{n}=C\left[\Phi_{n}(Z), \bar{G}\right] \rightarrow \infty$ as $n \rightarrow \infty$. There is no harm in assuming that $I_{n} \geq 1, n=1,2, \cdots$. Obviously $\tilde{\Phi}_{n}(Z)=\Phi_{n}(Z) / I_{n}$ satisfies the boundary conditions

$$
\begin{aligned}
& \operatorname{Re}\left[\overline{\tilde{\Lambda}_{n}(Z)} \tilde{\Phi}_{n}(Z)\right]=\left[\tilde{R}_{n}(Z)+\hat{H}(Z)\right] / I_{n}, Z \in \Gamma, \\
& \operatorname{Im}\left[\tilde{\Lambda}_{n}\left(\hat{a}_{n}\right) \tilde{\Phi}_{n}\left(\tilde{a}_{n}\right)\right]=\tilde{B}_{j n} / I_{n}, j \in J .
\end{aligned}
$$


Applying the Schwarz formula, the Cauchy formula and the method of symmetric extension (see Theorem 1.11, Chapter 1, [1]), the estimates

$$
C_{\eta \beta}\left[\tilde{\Phi}_{n}(Z), \overline{\hat{G}}\right] \leq M_{6}
$$

can be obtained, where $M_{6}=M_{6}(k, \beta, D)$. Thus we can select a subsequence of $\left\{\tilde{\Phi}_{n}(Z)\right\}$, which uniformly converge to an analytic function $\tilde{\Phi}_{0}(Z)$ in $G$, and $\tilde{\Phi}_{0}(Z)$ satisfies the homogeneous boundary conditions

$$
\operatorname{Re}\left[\overline{\tilde{\Lambda}_{0}(Z)} \Phi_{0}(Z)\right]=\tilde{H}(Z), z \in \Gamma, \operatorname{Im}\left[\overline{\tilde{\Lambda}_{0}\left(\tilde{a}_{j}\right)} \tilde{\Phi}_{0}\left(\tilde{a}_{j}\right)\right]=0, j \in J
$$

Duo to the uniqueness of solutions of Problem $\hat{B}$, we conclude that $\tilde{\Phi}_{0}(Z)=$ $0, Z \in \bar{G}$. However, from $C\left[\tilde{\Phi}_{n}(Z), \bar{G}\right]=1$, it follows that there exists a point $Z_{*} \in \bar{G}$, such that $\left|\tilde{\Phi}_{0}\left(Z_{*}\right)\right|=1$. This contradiction proves that (3.8) holds. Afterwards using the method which leads from $C\left[\tilde{\Phi}_{n}(Z), \bar{G}\right]=1$ to $(3.10)$, the estimate (3.7) can be derived.

Now we discuss the discontinuous Riemann-Hilbert boundary value problems (Problem B) for the elliptic complex equation (2.3) in the domain $D$, here we use the method of parameter extension.

Theorem 3.2. Suppose that the degenerate elliptic equation (2.3) satisfies the same conditions as in Theorem 3.1. Then Problem B for (2.3) has a continuous solution $W(Z)$ in $\bar{G}$.

Proof. We introduce the nonlinear elliptic complex equation with the parameter $t \in[0,1]$ :

$$
W_{\bar{Z}}-t F(Z, W)=E(Z), F(Z, W)=A(Z) W+B(Z) \bar{W}+C(Z),
$$

where $E(Z)$ is any measurable function in $G$ and $E(Z) \in L_{\infty}(D)$. When $t=0$, it is not difficult to see that there exists a unique solution $W(Z)$ of Problem $\mathrm{B}$ for the complex equation (3.11), which possesses the form

$$
\begin{gathered}
W(Z)=\Psi(Z)+\psi(Z), \\
\psi(z)=T E=-\frac{1}{\pi} \iint_{D} \frac{E(\zeta)}{H_{1}(\operatorname{Re} z(\zeta)) H_{2}(\operatorname{Im} z(\zeta))(\zeta-Z)} d \sigma_{\zeta}
\end{gathered}
$$

where $\Psi(z)$ is an analytic function in $D$ and satisfies the boundary conditions

$$
\begin{aligned}
& \operatorname{Re}[\overline{\Lambda(Z)} \Psi(Z)]=R(Z)-\operatorname{Re}[\overline{\Lambda(z)} \psi(Z)]+H(z), z \in \Gamma \\
& \operatorname{Im}\left[\overline{\Lambda\left(\hat{a}_{j}\right)} \Phi\left(\hat{a}_{j}\right)\right]=B_{j}-\operatorname{Im}\left[\overline{\Lambda\left(\hat{a}_{j}\right)} \psi\left(\hat{a}_{j}\right)\right], j \in J
\end{aligned}
$$

From Theorem 3.1, we see that $W(Z) \in B=C_{\beta}(\bar{D}), \beta(\leq \alpha)$ is a positive constant. Suppose that when $t=t_{0}\left(0 \leq t_{0}<1\right)$, Problem B for the complex 
equation (3.11) has a unique solution, we shall prove that there exists a neighborhood of $t_{0}: S=\left\{\left|t-t_{0}\right| \leq \delta, 0 \leq t \leq 1, \delta>0\right\}$, so that for every $t \in S$ and any function $E(Z) \in L_{\infty}(\bar{D})$, Problem B for (3.11) is solvable. In fact, the complex equation (3.11) can be written in the form

$$
W_{\bar{Z}}-t_{0} F\left(z, W, W_{Z}\right)=\left(t-t_{0}\right) F(Z, W)+E(Z) .
$$

We arbitrarily select a function $W_{0}(Z) \in B=C_{\beta}(\bar{D})$, in particular $W_{0}(Z)=0$ on $\bar{G}$. Let $W_{0}(Z)$ be replaced into the position of $W(Z)$ in the right hand side of (3.14). By Condition C, it is obvious that

$$
B_{0}(z)=\left(t-t_{0}\right) F\left(Z, W_{0}\right)+E(Z) \in L_{\infty}(\bar{D}) .
$$

Noting that (3.14) has a solution $W_{1}(Z) \in B$. Applying the successive iteration, we can find out a sequence of functions: $W_{n}(Z) \in B, n=1,2, \cdots$, which satisfy the complex equations

$$
W_{n+1 \bar{Z}}-t_{0} F\left(Z, W_{n+1}\right)=\left(t-t_{0}\right) F\left(Z, W_{n}\right)+E(Z), n=1,2, \cdots .
$$

The difference of the above equations for $n+1$ and $n$ is as follows:

$$
\begin{aligned}
& \left(W_{n+1}-W_{n}\right)_{\bar{Z}}-t_{0}\left[F\left(Z, W_{n+1}\right)-F\left(Z, W_{n}\right)\right] \\
& =\left(t-t_{0}\right)\left[F\left(Z, W_{n}\right)-F\left(Z, W_{n-1}\right)\right], n=1,2, \cdots .
\end{aligned}
$$

From Condition $\mathrm{C}$ and $(2.5)$ in $\bar{D}$, it can be seen that

$$
\begin{gathered}
F\left(Z, W_{n+1}\right)-F\left(Z, W_{n}\right) \\
=F\left(Z, W_{n+1}\right)-F\left(Z, W_{n+1}\right)+\left[F\left(Z, W_{n+1}\right)-F\left(Z, W_{n}\right)\right] \\
=A_{n+1}(Z)\left(W_{n+1}-W_{n}\right), A_{n+1}(Z) \in L_{\infty}(\bar{D}), n=1,2, \cdots,
\end{gathered}
$$

and

$$
\begin{gathered}
L_{\infty}\left[F\left(Z, W_{n}\right)-F\left(Z, W_{n-1}\right), \bar{D}\right] \leq k_{0} C\left[W_{n}-W_{n-1}, \bar{D}\right] \\
\leq k_{0} C_{\beta}\left[W_{n}-W_{n-1}, \bar{D}\right]=k_{0} L_{n} .
\end{gathered}
$$

Moreover, $W_{n+1}(z)-W_{n}(z)$ satisfies the homogeneous boundary conditions

$$
\begin{aligned}
& \operatorname{Re}\left[\overline{\lambda(z)}\left(W_{n+1}(Z)-W_{n}(Z)\right)\right]=\hat{H}(Z), Z \in \Gamma, \\
& \operatorname{Im}\left[\overline{\Lambda\left(\hat{a}_{j}\right)}\left(W_{n+1}\left(\hat{a}_{j}\right)-W_{n}\left(\hat{a}_{j}\right)\right)\right]=0, j \in J .
\end{aligned}
$$

On the basis of Theorem 3.1, we have

$$
L_{n+1}=C_{\beta}\left[W_{n+1}-W_{n}, \bar{G}\right] \leq M_{7}\left|t-t_{0}\right| k_{0} L_{n},
$$


where $M_{7}=M_{7}(k, \beta, D)$ is a positive constant. Provided $\delta(>0)$ is small enough, so that $\eta=\delta M_{7} k_{0}<1$, it can be obtained that

$$
L_{n+1} \leq \eta L_{n} \leq \eta^{n} L_{1}=\eta^{n}\left[C_{\beta}\left(W_{1}, \bar{D}\right)\right.
$$

for every $t \in E$. Thus

$$
\begin{gathered}
S\left(W_{n}-W_{m}\right)=C_{\beta}\left[W_{n}-W_{m}, \bar{D}\right] \\
\leq L_{n}+L_{n-1}+\cdots+L_{m+1} \leq\left(\eta^{n-1}+\eta^{n-2}+\cdots+\eta^{m}\right) L_{1} \\
=\eta^{m}\left(1+\eta+\cdots+\eta^{n-m-1}\right) L_{1} \leq \eta^{N+1} \frac{1-\eta^{n-m}}{1-\eta} L_{1} \leq \frac{\eta^{N+1}}{1-\eta} L_{1}
\end{gathered}
$$

for $n \geq m>N$, where $N$ is a positive integer. This shows that $S\left(W_{n}-W_{m}\right) \rightarrow 0$ as $n, m \rightarrow \infty$. Following the completeness of the Banach space $B=C_{\beta}(\bar{D})$, there is a function $w_{*}(z) \in B$, such that when $n \rightarrow \infty$,

$$
S\left(W-W_{*}\right)=C_{\beta}\left[W_{n}-W_{*}, \bar{D}\right] \rightarrow 0 .
$$

By Condition $\mathrm{C}$ and (2.4), from (3.1) it follows that $W_{*}(z)$ is a solution of Problem B for (3.15), i.e. (3.11) for $t \in E$. It is easy to see that the positive constant $\delta$ is independent of $t_{0}\left(0 \leq t_{0}<1\right)$. Hence from Problem $\mathrm{B}$ for the complex equation (3.15) with $t=t_{0}=0$ is solvable, we can derive that when $t=\delta, 2 \delta, \cdots,[1 / \delta] \delta, 1$, Problem B for (3.15) are solvable, especially Problem B for (3.15) with $t=1$ and $E(Z)=0$, namely Problem B for (2.3) has a unique solution.

Theorem 3.3. Suppose that Problem A of the complex equation (1.3) satisfies Condition $\mathrm{C}$ and (1.4), (1.8). Then Problem A for (1.3) has a continuous $w(z)$ in $\bar{D}$.

Proof. On the basis of Theorem 3.2, we know that Problem B for (2.3) has a continuous solution $W(Z)$ in $G$. Moreover by Theorem 2.1, it is easy to see that $w(z)=W[Z(z)]$ is just a solution of Problem A for (1.3).

\section{Hölder Continuity of A Singular Double Integral}

It is clear that the complex equation

$$
W_{\bar{Z}}=g(Z) \text { in } \overline{D_{Z}}
$$


is a special case of equation (2.3), where $D_{Z}$ is a bounded multiply connected domain with the boundary $\partial D \in C_{\mu}^{1}(0<\mu<1)$.

Now we consider the function $g(Z) \in L_{\infty}\left(D_{Z}\right)$, and first extend the function $g(Z)$ to the exterior of $\overline{D_{Z}}$ in $\mathbb{C}$, i.e. set $g(Z)=0$ in $\mathbb{C} \backslash \overline{D_{Z}}$, hence we can only discuss the domain $D_{0}=\left[\left\{|X|<R_{0}\right\} \cup\left\{|Y|<R_{0}\right\}\right] \supset \overline{D_{Z}}$, here $Z=X+i Y, R_{0}$ is an appropriately large positive number. In the following we shall verify that the integral

$$
\begin{gathered}
\Psi(Z)=T\left(\frac{g}{H_{1} H_{2}}\right)=-\frac{1}{\pi} \iint_{D_{0}} \frac{g(t)}{(t-Z) H_{1}(\operatorname{Re} z(t)) H_{2}(\operatorname{Im} z(t))} d \sigma_{t} \text { in } D_{0}, \\
L_{\infty}\left[g(Z), D_{0}\right] \leq k_{3}
\end{gathered}
$$

satisfies the estimate (4.3) below, in which $m=m_{1} /\left(1+m_{1}-m_{3}\right)+m_{2} /\left(1+m_{2}-\right.$ $\left.m_{4}\right)<1$ is as stated in Section 1 , and $H_{j}(x)=H_{j}[\operatorname{Re} z(Z)](j=1,3), H_{j}(y)=$ $H_{j}[\operatorname{Im} z(Z)](j=2,4), z(Z)$ is as stated in (2.1). It is clear that the function

$$
g(Z) H_{3} H_{4} / H_{1}(x) H_{2}(y)=g(Z) H_{3}[\operatorname{Re} z(Z)] H_{4}[\operatorname{Im} z(Z)] / H_{1}[\operatorname{Re} z(Z)] H_{2}[\operatorname{Im} z(Z)]
$$

belongs to the space $L_{1}\left(D_{0}\right)$ and in general is not belonging to the space $L_{p}\left(D_{0}\right)(p>2)$, and the integral $\Psi\left(Z_{0}\right)$ is definite when $\operatorname{Im} Z_{0} \neq 0$. If $Z_{0} \in D_{0}$ and $\operatorname{Im} Z_{0}=0$, we can define the integral $\Psi\left(Z_{0}\right)$ as the limit of the corresponding integral over $D_{0} \cap\left\{\left|\operatorname{Re} t-\operatorname{Re} Z_{0}\right| \geq \varepsilon\right\} \cup\left\{\left|\operatorname{Im} t-\operatorname{Im} Z_{0}\right| \geq \varepsilon\right\}$ as $\varepsilon \rightarrow 0$, where $\varepsilon$ is a sufficiently small positive number. The Hölder continuity of the singular integral will be proved by the following method.

Theorem 4.1. If the function $g(Z)$ in $D_{Z}$ satisfies the condition in (4.2), and $H_{j}(x)=|x|^{m_{j}} h_{j}(x)(j=1,3), H_{j}(y)=x^{m_{j}} h_{j}(y)(j=2,4)$, in which $m_{j}\left(j=1,2,3,4, m_{3} \leq \min \left(1, m_{1}\right), m_{4} \leq \min \left(1, m_{2}\right)\right)$ are positive numbers satisfying the condition $m_{1} /\left(m_{1}-m_{3}+1\right)+m_{2} /\left(m_{2}-m_{4}+1\right)<1, h_{1}(x), h_{2}(y)$ area continuous positive functions, then the integral in (4.2) satisfies the estimate

$$
C_{\beta}\left[\Psi(Z), \overline{D_{Z}}\right] \leq M_{1},
$$

where $\beta=1-m_{1} /\left(m_{1}-m_{3}+1\right)-m_{2} /\left(m_{2}-m_{4}+1\right)-\delta=1-m-\delta, \delta$ is a sufficiently small positive constant, and $M_{1}=M_{1}\left(\beta, k_{3}, H, D_{Z}\right)$ is a positive constant, here $H=H\left(H_{1}, H_{2}, H_{3}, H_{4}\right)$.

Proof. We first give the estimates of $\Psi(Z)$ of (4.2) in $D \cap\{X \geq 0\} \cap\{Y \geq$ $0\}$, and verify the boundedness of the function in (4.2). As stated Section 1 , we have $H_{1}(x) / H_{3}(x) \geq s X^{m_{1} /\left(m_{1}-m_{2}+1\right)}, H_{2}(y) / H_{4}(y) \geq s Y^{m_{2} /\left(m_{2}-m_{4}+1\right)}$, where $s$ is a positive constant. For any two points $Z_{0}=X_{0} \in \gamma=[0,1)$ on $X$-axis and $Z_{1}=X_{1}+i Y_{1}\left(X_{1}>0, Y_{1}>0\right) \in D_{0}$ satisfying the condition 
$2 \operatorname{Im} Z_{1} / \sqrt{3} \leq\left|Z_{1}-Z_{0}\right| \leq 2 \operatorname{Im} Z_{1}$, this means that the inner angle at $Z_{0}$ of the triangle $Z_{0} Z_{1} Z_{2}\left(Z_{2}=X_{0}+i Y_{1} \in D_{0}\right)$ is not less than $\pi / 6$ and not greater than $\pi / 3$, choose a sufficiently large positive number $q$, from the Hölder inequality, we have $L_{1}\left[\Psi(t), D_{0}\right] \leq L_{q}\left[g(t), D_{0}\right] L_{p}\left[1 / H_{1}\left(\operatorname{Re} z(t) H_{2}(\operatorname{Im} z(t))(t-Z), D_{0}\right]\right.$, in which $p=q /(q-1)(>1)$ is close to 1 . In fact we can derive as follows

$$
\begin{gathered}
\left|\Psi\left(Z_{0}\right)\right| \leq\left|\frac{1}{\pi} \iint_{D_{0}} \frac{g(t)}{\left(t-Z_{0}\right) H_{1}(\operatorname{Re} z(t)) H_{2}(\operatorname{Im} z(t))} d \sigma_{t}\right| \\
\leq \frac{1}{s \pi} L_{q}\left[g(Z), D_{0}\right]\left[\iint_{D_{0}}\left|\frac{1}{t^{m}\left(t-Z_{0}\right)}\right|^{p} d \sigma_{t}\right]^{1 / p}=\frac{1}{s \pi} L_{q}\left[g(Z), D_{0}\right] J_{1}^{1 / p},
\end{gathered}
$$

where

$$
\begin{aligned}
J_{1} & =\iint_{G_{0}}\left|\frac{1}{t^{m}\left(t-Z_{0}\right)}\right|^{p} d \sigma_{t} \\
& \leq \iint_{G_{0}} \frac{1}{|t|^{p m}\left|\operatorname{Re}\left(t-Z_{0}\right)\right|^{p \beta_{0}}\left|\operatorname{Im}\left(t-Z_{0}\right)\right|^{p\left(1-\beta_{0}\right)}} d \sigma_{t} \\
& \leq\left|\int_{0}^{d_{0}} \frac{\left|Y-Y_{0}\right|^{p \beta_{0}}}{Y^{p m}} d Y \int_{d_{1}}^{d_{2}} \frac{1}{\left|X-X_{0}\right|^{p\left(1-\beta_{0}\right)}} d X\right| \leq k_{4},
\end{aligned}
$$

where $d_{0}=\max _{Z \in \overline{G_{0}}} \operatorname{Im} Z, d_{1}=\min _{Z \in \overline{G_{0}}} \operatorname{Re} Z, d_{2}=\max _{Z \in \overline{G_{0}}} \operatorname{Re} Z, \beta_{0}=1-m-\varepsilon$, $\varepsilon$ is a sufficiently small positive constant, we can choose $\varepsilon=2(p-1) / p$, such that $p\left(1-\beta_{0}\right)<1$ and $p\left[m+\beta_{0}\right]<1$, and $k_{4}=k_{4}\left(\beta, k_{3}, H, G_{0}\right)$ is a nonnegative constant.

Next we estimate the Hölder continuity of the integral $\Psi(Z)$ in $\overline{G_{0}}$, i.e.

$$
\begin{gathered}
\left|\Psi\left(Z_{1}\right)-\Psi\left(Z_{0}\right)\right| \leq \frac{\left|Z_{1}-Z_{0}\right|}{\pi}\left|\iint_{G_{0}} \frac{g(t)}{\left(t-Z_{0}\right)\left(t-Z_{1}\right) H_{1}(\operatorname{Re} z(t)) H_{2}(\operatorname{Im} z(t))} d \sigma_{t}\right| \\
\leq \frac{\left|Z_{1}-Z_{0}\right|}{s \pi} L_{q}\left[g(Z), G_{0}\right]\left[\iint_{G_{0}}\left|\frac{1}{t^{m}\left(t-Z_{0}\right)\left(t-Z_{1}\right)}\right|^{p} d \sigma_{t}\right]^{1 / p},
\end{gathered}
$$

and 


$$
\begin{aligned}
J_{2}= & \iint_{G_{0}}\left|\frac{1}{t^{m}\left(t-Z_{0}\right)\left(t-Z_{1}\right)}\right|^{p} d \sigma_{t} \\
\leq & \iint_{G_{0}} \frac{\left|\operatorname{Re}\left(t-Z_{0}\right)\right|^{p\left(\beta_{0} / 2-1\right)}\left|\operatorname{Re}\left(t-Z_{1}\right)\right|^{p\left(\beta_{0} / 2-1\right)}}{|t|^{p m}\left|\operatorname{Im}\left(t-Z_{0}\right)\right|^{p \beta_{0} / 2}\left|\operatorname{Im}\left(t-Z_{1}\right)\right|^{p \beta_{0} / 2}} d \sigma_{t} \\
\leq & \int_{0}^{d_{0}} \frac{1}{Y^{p m}\left|\operatorname{Im}\left(Y-Z_{0}\right)\right|^{p \beta_{0} / 2}\left|\operatorname{Im}\left(Y-Z_{1}\right)\right|^{p \beta_{0} / 2}} d Y \\
& \times \int_{d_{1}}^{d_{2}} \frac{1}{\left|\operatorname{Re}\left(t-Z_{0}\right)\right|^{p\left(1-\beta_{0} / 2\right)}\left|\operatorname{Re}\left(t-Z_{1}\right)\right|^{p\left(1-\beta_{0} / 2\right)}} d X, \\
\leq & k_{5} \int_{d_{1}}^{d_{2}} \frac{1}{\left.\mid X-X_{0}\right)\left.\right|^{p\left(1-\beta_{0} / 2\right)}\left|X-X_{1}\right|^{p\left(1-\beta_{0} / 2\right)}} d X
\end{aligned}
$$

where $\beta_{0}=1-m-\varepsilon$ is chosen as before and

$$
k_{5}=\max _{Z_{0}, Z_{1} \in G_{0}} \int_{0}^{d_{0}} \frac{1}{Y^{p m}\left|\operatorname{Im}\left(Y-Z_{0}\right)\right|^{p \beta_{0} / 2}\left|\operatorname{Im}\left(Y-Z_{1}\right)\right|^{p \beta_{0} / 2}} d Y .
$$

Denote $\rho_{0}=\left|\operatorname{Re}\left(Z_{1}-Z_{0}\right)\right|=\left|X_{1}-X_{0}\right|, L_{1}=G_{0} \cap\left\{\left|X-X_{0}\right| \leq 2 \rho_{0}, Y=Y_{0}\right\}$ and $L_{2}=G_{0} \cap\left\{2 \rho_{0}<\left|X-X_{0}\right| \leq 2 \rho_{1}<\infty, Y=Y_{0}\right\} \supset\left[d_{1}, d_{2}\right] \backslash L_{1}$, where $\rho_{1}$ is a sufficiently large positive number, we can derive

$$
\begin{aligned}
J_{2} \leq & k_{5}\left[\int_{L_{1}} \frac{1}{\left|X-X_{0}\right|^{p\left(1-\beta_{0} / 2\right)}\left|X-X_{1}\right|^{p\left(1-\beta_{0} / 2\right)}} d X\right. \\
& \left.+\int_{L_{2}} \frac{1}{\left|X-X_{0}\right|^{p\left(1-\beta_{0} / 2\right)}\left|X-X_{1}\right|^{p\left(1-\beta_{0} / 2\right)}} d X\right] \\
\leq & k_{5}\left[\left|X_{1}-X_{0}\right|^{1-2 p+p \beta_{0}} \int_{|\xi| \leq 2} \frac{1}{|\xi|^{p\left(1-\beta_{0} / 2\right)}|\xi \pm 1|^{p\left(1-\beta_{0} / 2\right)}} d \xi\right. \\
& \left.+k_{6}\left|\int_{2 \rho_{0}}^{2 \rho_{1}} \rho^{p \beta_{0}-2 p} d \rho\right|\right] \leq k_{7}\left|X_{1}-X_{0}\right|^{1-p\left(2-\beta_{0}\right)}= \\
= & k_{7}\left|X_{1}-X_{0}\right|^{p(1-m-\varepsilon+1 / p-2)}
\end{aligned}
$$

where we use $\left|X-X_{0}\right|=\xi\left|X_{1}-X_{0}\right|,\left|X-X_{1}\right|=\left|X-X_{0}-\left(X_{1}-X_{0}\right)\right|=|\xi \pm 1|\left|X_{1}-X_{0}\right|$ if $X \in L_{1},\left|X-X_{0}\right|=\rho \leq 2\left|X-X_{1}\right|$ if $X \in L_{2}$, choose that $p(>1)$ is close to 1 such that $1-p\left(2-\beta_{0}\right)<0$, and $k_{j}=k_{j}\left(\beta, k_{3}, H, G_{0}\right)(j=6,7)$ are non-negative constants. Thus we get

$$
\left|\Psi\left(Z_{1}\right)-\Psi\left(Z_{0}\right)\right| \leq k_{7}\left|Z_{1}-Z_{0}\right|\left|X_{1}-X_{0}\right|^{1-m-\varepsilon+1 / p-2} \leq k_{8}\left|Z_{1}-Z_{0}\right|^{\beta},
$$

in which we use that the inner angle at $Z_{0}$ of the triangle $Z_{0} Z_{1} Z_{2}\left(Z_{2}=X_{0}+\right.$ $\left.i Y_{1} \in G_{0}\right)$ is not less than $\pi / 6$ and not greater than $\pi / 3$, and choose $\varepsilon=$ 
$2(p-1) / p, \beta=1-m-\delta, \delta=3(p-1) / p, k_{8}=k_{8}\left(\beta, k_{3}, H, G_{0}\right)$ is a non-negative constant. The above points $Z_{0}=X_{0}, Z_{1}=X_{1}+i Y_{1}$ can be replaced by $Z_{0}=$ $X_{0}+i Y_{0}, Z_{1}=X_{1}+i Y_{1} \in \overline{G_{0}}, 0<Y_{0}<Y_{1}$ and $2\left(Y_{1}-Y_{0}\right) / \sqrt{3} \leq\left|Z_{1}-Z_{0}\right| \leq$ $2\left(Y_{1}-Y_{0}\right)$. In addition we consider any two points $Z_{1}=X_{1}+i Y_{1}, Z_{2}=X_{2}+i Y_{1}$ and $X_{1}<X_{2}$, from the above estimates, the following estimate can be derived

$$
\begin{gathered}
\left|\Psi\left(Z_{1}\right)-\Psi\left(Z_{2}\right)\right| \leq\left|\Psi\left(Z_{1}\right)-\Psi\left(Z_{3}\right)\right|+\left|\Psi\left(Z_{3}\right)-\Psi\left(Z_{2}\right)\right| \\
\leq k_{8}\left|Z_{1}-Z_{3}\right|^{\beta}+k_{8}\left|Z_{3}-Z_{2}\right|^{\beta} \leq k_{9}\left|Z_{1}-Z_{2}\right|^{\beta},
\end{gathered}
$$

where $Z_{3}=\left(X_{1}+X_{2}\right) / 2+i\left[Y_{1}+\left(X_{2}-X_{1}\right) /(2 \sqrt{3})\right]$. If $Z_{1}=X_{1}+i Y_{1}, Z_{2}=$ $X_{1}+i Y_{2}, Y_{1}<Y_{2}$, and we choose $Z_{3}=X_{1}+\left(Y_{2}-Y_{1}\right) / 2 \sqrt{3}+i\left(Y_{2}+Y_{1}\right) / 2$, and can also get (4.7). If $Z_{1}=X_{1}+i Y_{1}, Z_{2}=X_{2}+i Y_{2}, X_{1}<X_{2}, Y_{1}<Y_{2}$, and we choose $Z_{3}=X_{2}+i Y_{1}$, obviously

$$
\left|\Psi\left(Z_{1}\right)-\Psi\left(Z_{2}\right)\right| \leq\left|\Psi\left(Z_{1}\right)-\Psi\left(Z_{3}\right)\right|+\left|\Psi\left(Z_{3}\right)-\Psi\left(Z_{2}\right)\right|,
$$

and $\left|\Psi\left(Z_{1}\right)-\Psi\left(Z_{3}\right)\right|,\left|\Psi\left(Z_{3}\right)-\Psi\left(Z_{2}\right)\right|$ can be estimated by the above way, hence we can obtain the estimate of $\left|\Psi\left(Z_{1}\right)-\Psi\left(Z_{2}\right)\right|$. We can similarly discuss for any two points $Z_{0}=X_{0} \in \gamma_{1}=[0,1)$ on $X$-axis or $Z_{0}=Y_{0} \in \gamma_{2}=[0,1)$ on $Y$-axis.

For the function $\Psi(Z)$ of (4.2) in $G_{0} \cap\{Y \leq 0\} \cap\{X \geq 0\}$ and $G_{0} \cap\{X \leq 0\}$, the similar estimates can be also derived. Hence we have the estimate (4.3).

\section{Acknowledgments}

The research was supported by PHR (IHLB 201106206), and College Students Scientific Research and Undertaking Starting Action Project (SJ201301016).

\section{References}

[1] I.N. Vekua, Generalized Analytic Functions, Pergamon, Oxford (1962).

[2] A.V. Bitsadze, Some Classes of Partial Differential Equations, Gordon and Breach, New York (1988).

[3] V. Gutlyanskii, V. Ryazanov, U. Srebro, E. Yakubov, The Beltrami equations, A geometric Approach, Springer Verlag, Heidelberg (2012).

[4] B. Bojarski, V. Gutlyanskii, V. Ryazanov, Dirichlet problem for general Beltrami equation in Jordan domains, Ukrainian Mathematical Sciences, 9 (2012), 460-476. 
[5] Yu. Dybov, On regular solutions of the Dirichlet problem for the Beltrami equations, Complex Variables and Elliptic Equations. 55 (2010), 1099-1116.

[6] D.A. Kovtonyuk, I.V. Petkov, V.I. Ryazanov, On the Dirichlet problem for the Beltrami equations in finitely connected domains, Ukrainian Mathematical Journal. 64 (2012), 1064-1077.

[7] G.C. Wen, H. Begehr, Boundary value problems for elliptic equations and systems, Longman Scientific and Technical Company, Harlow (1990).

[8] G.C. Wen, Conformal Mappings and Boundary Value Problems, Translations of Mathematics Monographs 106, Amer. Math. Soc., Providence, RI (1992).

[9] G.C. Wen, Linear and Nonlinear Elliptic Complex Equations, Shanghai Scientific and Technical Publishers, Shanghai (1986) (Chinese).

[10] G.C. Wen, C.W., M.Y. Tian, Function Theoretic Methods of Free Boundary Problems and Their Applications to Mechanics, Higher Education Press, Beijing (1996) (Chinese).

[11] G.C. Wen, Recent Progress in Theory and Applications of Modern Complex Analysis, Science Press, Beijing (2010).

[12] G.C. Wen, Approximate Methods and Numerical Analysis for Elliptic Complex Equations, Gordon and Breach, Amsterdam (1999).

[13] G.C. Wen, Linear and nonlinear parabolic complex equations, World Scientific Publishing Co., Singapore (1999).

[14] G.C. Wen, B.T. Zou, Initial-boundary value problems for nonlinear parabolic equations in higher dimensional domains, Science Press, Beijing (2002).

[15] G.C. Wen, Linear and quasilinear complex equations of hyperbolic and mixed Type, Taylor \& Francis, London (2002).

[16] S. Huang, Y.Y. Qiao, G.C. Wen, Real and complex Clifford analysis, Springer-Verlag, Heidelberg (2005).

[17] G.C. Wen, Nonlinear partial differential complex equations, Science Press, Beijing (1999, Chinese). 
[18] G.C. Wen, D.C. Chen, Z.L. Xu, Nonlinear complex analysis and its applications, Mathematics Monograph Series 12, Science Press, Beijing, (2008). 